\begin{abstract}
Feature distinctiveness effects in language acquisition and lexical processing:
Insights from megastudies
\end{abstract}

\author{
Cynthia S. Q. Siew ${ }^{1}$
}

${ }^{1}$ Department of Psychology, National University of Singapore

Corresponding Author:

Cynthia S. Q. Siew, PhD

Department of Psychology

9 Arts Link, Block AS4 \#02-23

National University of Singapore

Singapore 117570, Singapore

Email: cynthia@nus.edu.sg 


\begin{abstract}
Semantic features are central to many influential theories of word meaning and semantic memory, but new methods of quantifying the information embedded in feature production norms are needed to advance our understanding of semantic processing and language acquisition. This paper capitalized on databases of semantic feature production norms and age-of-acquisition ratings, and megastudies including the English Lexicon Project and the Calgary Semantic Decision Project, to examine the influence of feature distinctiveness on language acquisition, visual lexical decision, and semantic decision. A feature network of English words was constructed such that edges in the network represented feature distance, or dissimilarity, between words (i.e., Jaccard and Manhattan distances of probability distributions of features elicited for each pair of words), enabling us to quantify the relative feature distinctiveness of individual words relative to other words in the network. Words with greater feature distinctiveness tended to be acquired earlier. Regression analyses of megastudy data revealed that Manhattan feature distinctiveness inhibited performance on the visual lexical decision task, facilitated semantic decision performance for concrete concepts, and inhibited semantic decision performance for abstract concepts. These results demonstrate the importance of considering the structural properties of words embedded in a semantic feature space in order to increase our understanding of semantic processing and language acquisition.
\end{abstract}

Key words: feature distinctiveness, feature production norms, megastudies, lexical decision, semantic decision task, age of acquisition 


\section{Feature distinctiveness effects in language acquisition and lexical processing: Insights from megastudies}

What gives a word its meaning? The lack of a straightforward answer to this question demonstrates the complexity of quantifying semantic information associated with words. There are many approaches to quantify the semantics of words, from computing co-occurrence statistics in natural language (Bullinaria \& Levy, 2007; Lund \& Burgess, 1996), compiling databases of free associations (De Deyne, Navarro, Perfors, Brysbaert, \& Storms, 2018; De Deyne \& Storms, 2008; Nelson, McEvoy, \& Schreiber, 2004), and eliciting features associated with words (McRae, Cree, Seidenberg, \& McNorgan, 2005; Vinson \& Vigliocco, 2008). Although these approaches are different, they share one central idea - that the meaning of a word can be understood through the relationships a particular word maintains with other words (Firth, 1957). For instance, words that co-occur in similar contexts are semantically similar to each other (Bullinaria \& Levy, 2007), and words that elicit similar associations or features are considered to be semantically similar to each other (De Deyne et al., 2018; Nelson et al., 2004). Hence, much research in semantic memory and conceptual representations is devoted to developing methods that quantify the semantic "spaces" that words are found in.

Semantic features are central to many theories and models of semantic memory and category learning, and features are commonly used as representations of concepts, objects, and words (Collins \& Loftus, 1975; Minda \& Smith, 2002; Plaut \& Shallice, 1993; Sizemore, Karuza, Giusti, \& Bassett, 2018). Data from feature production norms, where participants list features of a given concept, provides the empirical foundation against which theories of conceptual and semantic representation can be tested. In this paper we investigate the semantic feature spaces of words, and examine how feature distinctiveness, a semantic measure that 
measures how distinctive a word is relative to other words based on their semantic features, influences lexical and semantic processing.

At this point, it is important to note that the way in which feature distinctiveness is defined in the present manuscript is conceptually different from the notion of distinctive features. In the literature, distinctive features are frequently defined as features that have high cue validity for a given concept (e.g., the feature $<$ moo $>$ is distinctive as it cues the concept $<$ cow $>$ ). The distinctiveness of a feature is typically inferred via an analysis of feature production norms, and features that occur in few concepts and help people discriminate among similar concepts are said to be distinctive (Cree, McNorgan, \& McRae, 2006; Garrard, Lambon Ralph, Hodges, \& Patterson, 2001; Taylor, Salamoura, Randall, Moss, \& Tyler, 2008). Furthermore, distinctive features appear to play an important role in the computation of a word's meaning, as evidenced from behavioral work involving speeded feature verification and sentence completion, and computational simulations of connectionist models of semantic memory, as well as research on patients with semantic-category deficits that suggest that distinctive features (particularly if they are not highly correlated with other features) are less robust and tend to be lost first in cases of acquired brain damage (Cree et al., 2006; Cree \& McRae, 2003; Garrard et al., 2001; Moss, Tyler, \& Jennings, 1997; Randall, Moss, Rodd, Greer, \& Tyler, 2004; Tyler \& Moss, 2001; Tyler, Moss, Durrant-Peatfield, \& Levy, 2000). However, in all of the abovementioned research, the emphasis has been on quantifying the internal structure of the concepts; for instance, the features they possess, how these features are (more or less) correlated with each other among concepts of similar or different domains (Tyler \& Moss, 2001). A different approach is adopted in the present manuscript. Instead of focusing on the characteristics of the features or the internal semantic properties of concepts, we use features that are elicited by concepts in a feature 
production task to quantify how distinctive words (i.e., concepts or the cues in the feature production task) are relative to other words in a global feature semantic space (i.e., a word's feature distinctiveness). This is a novel perspective on semantic memory because it attempts to bring together two key ideas in competing theoretical approaches to semantic memory - that the meaning of a word (i) can be decomposed into smaller units (i.e., decompositional or componential theories; Rips, Shoben, \& Smith, 1973), and (ii) depends on its position in a broader semantic network or space (i.e., semantic network models; Collins \& Loftus, 1975). This paper puts forward the notion that the internal properties of words (i.e., their semantic features) plays a part in determining where words are located in an external, semantic feature space, and tests the hypothesis that a word's distinctiveness in that external, semantic feature space has implications for semantic processing.

Not only have feature production norms been used to define the semantics of words, these data also permit more detailed investigations into the way that semantic features of objects drive vocabulary acquisition and category learning among children (Collins \& Loftus, 1975; Minda \& Smith, 2002; Plaut \& Shallice, 1993; Sizemore, Karuza, Giusti, \& Bassett, 2018). The analyses conducted by Engelthaler and Hills (2017) are particularly relevant to the present paper. The authors used feature network analysis to study how feature distinctiveness of a concept affected word learning and language acquisition. In their analyses, a network representation was used to define the semantic space, such that each concept was treated as a node in the feature network, and connections between concepts represented distances in the semantic feature space. This allowed them to quantify the feature distinctiveness of a word relative to other words in the network. Engelthaler and Hills (2017) found that distinctive objects tended to be acquired early in life, pushing forward an intriguing hypothesis about how the features of objects might drive 
the underlying mechanism by which the mutual exclusivity bias, that is, the tendency to pair one object with a single label (Jaswal \& Hansen, 2006; Markman \& Wachtel, 1988), enhances word learning. Specifically, two highly dissimilar objects are less likely to be assigned to the same label as compared to two similar objects, such that distinctive objects are more likely to acquire new labels and have lower age-of-acquisition ratings.

Given that the features of concepts and objects play a key role in language acquisition (e.g., Smith et al., 2002), it is perhaps not surprising that features also influence lexical processing. Indeed, a growing body of evidence in psycholinguistics shows that a host of semantic variables influences lexical and semantic processing in adults and children (Hsiao \& Nation, 2018; Pexman, Hargreaves, Siakaluk, Bodner, \& Pope, 2008; Pexman, Holyk, \& Monfils, 2003; Recchia \& Jones, 2012; Tousignant \& Pexman, 2012; Yap, Tan, Pexman, \& Hargreaves, 2011). These semantic variables include semantic neighbors based on word cooccurrences, number of features, and contextual dispersion (i.e., the number of contexts that a word is found in; Adelman, Brown, \& Quesada, 2006). Specifically, the number-of-features measure facilitated word recognition and semantic classification—words with more features are said to have more robust semantic representations making them more quickly and accurately recognized in a number of tasks (Pexman et al., 2008; Yap et al., 2011).

However, in the previous work, the most common way of quantifying the semantic properties of individual words based on semantic features is to simply count the number of features associated with a given word (e.g., Pexman et al., 2003). This measure does not consider that some features are listed more frequently than others, that words can share similar features (Hills, Maouene, Maouene, Sheya, \& Smith, 2009; Sizemore et al., 2018), and that distinctive features can help distinguish between words (Marques, 2005). The number-of-features measure 
treats each feature associated with a word as an independent piece of information, when in actuality words are semantically related to other words based on their shared and distinctive features within a highly complex, multi-dimensional semantic structure (Sizemore et al., 2018; Steyvers \& Tenenbaum, 2005). Indeed, structural properties of words in language networks, where connections between words represent form-based similarity or semantic relationships, have been shown to influence many aspects of word learning (Goldstein \& Vitevitch, 2014), word recognition (Siew \& Vitevitch, 2019), and production (Castro \& Stella, 2019; see Siew, Wulff, Beckage, \& Kenett, 2019, for a review). Given the body of research showing that the phonological and semantic structure of words in the lexicon has demonstrable influences on lexical processing, this suggests that it is important to consider the structure of words in a semantic feature network when examining the influence of semantic features on word processing.

Fortunately, the approach taken by Engelthaler and Hills (2017) provides us with a way to quantify the structure of the semantic feature network. Feature network analysis allows us to quantify the distinctiveness of words relative to other words based on their features. In addition, there now exist a number of databases that permit a large-scale investigation into these questions - specifically, feature production norms for 4,436 words from Buchanan, Valentine, and Maxwell (2019), the Calgary semantic decision project which has semantic decision latencies and accuracies for 10,000 words (Pexman, Heard, Lloyd, \& Yap, 2017), and the English Lexicon Project which has lexical decision latencies and accuracies for thousands of words (Balota et al., 2007). Indeed, analyzing megastudy data could allow one to make a more realistic assumptions about semantic processing in the general population. However, it is important to note that subsequent analysis of feature distinctiveness (and any other semantic or 
lexical variable) on processing could be affected by the word stimuli included in the behavioral testing and the number of participants enrolled in the data collection. In the present paper, we investigate the influence of semantic feature distinctiveness, as quantified by distances between words in a semantic feature network, on word recognition.

Although we expect to find effects of feature distinctiveness in word processing, it is not immediately clear what the directionality of this effect might be. One might hypothesize that when a word is activated, this will also activate its features (commonly implemented in connectionist models of semantic memory; e.g., Cree et al., 2006; Plaut \& Shallice, 1993; Randall et al., 2004). Within a spreading activation framework where activation can spread among related words (Collins \& Loftus, 1975; Siew, 2019), it is plausible to assume that activation tends to spread among words that share similar features, but less so among words that do not share similar features. Therefore, words with low feature distinctiveness would tend to activate many other words in the network (due to low feature distances). This might facilitate word recognition in a lexical decision task (e.g., faster reaction time) because the activation of several lexical nodes might provide more "evidence" for the presence of a word. On the other hand, words with high feature distinctiveness would activate few words (due to high feature distances), such that there are fewer competing words in the lexical system and enhances performance in the semantic decision task that emphasizes semantic processing.

This paper capitalizes on the availability of these databases, as well as quantitative tools such as network analysis and methods for computing distances between words, to achieve the following goals. First, it will attempt to replicate Engelthaler and Hills (2017)'s finding that words with greater feature distinctiveness tend to be acquired early in life (i.e., have lower age of acquisition ratings) with a larger set of words for which feature production norms are now 
available. Second, a series of analyses will be conducted on megastudy data to see if feature distinctiveness influences lexical and semantic processing among adults.

\section{Method}

\section{Feature production norms}

We used semantic feature production norms compiled and maintained by Buchanan et al. (2019), available at https://osf.io/cjyzw/. The data was obtained via a feature listing or production task, where participants provided lists of features for each concept (i.e., cue word) presented. The Buchanan et al. database contains data for 4,436 English words with features collected from almost 200 adult participants, and is supplemented with data from previous feature norm databases (Buchanan, Holmes, Teasley, \& Hutchison, 2013; Devereux, Tyler, Geertzen, \& Randall, 2014; McRae et al., 2005; Vinson \& Vigliocco, 2008). There was a total of 3,981 unique features. Table 1 shows the distribution of word classes across all cue-feature pairs in the feature production norms $(N=69,284)$. It can be observed that although a large proportion of cue-feature pairs are between nouns $(\sim 46 \%)$, a variety of word classes was represented in the feature production norms. In the present analysis, all features were included in the computation of feature distinctiveness, and the word class of cues were included as covariates in the subsequent regression analysis.

Table 1. Distribution of word classes across cue-feature pairs in the Buchanan et al. (2019) feature production norms $(N=69,284)$

\begin{tabular}{cccccc}
\hline & & \multicolumn{4}{c}{ Feature } \\
\cline { 3 - 6 } & & Noun & Verb & Adjective & Other \\
\hline \multirow{2}{*}{ Cue } & Noun & $46.43 \%$ & $15.91 \%$ & $11.03 \%$ & $1.57 \%$ \\
& Verb & $6.03 \%$ & $4.39 \%$ & $1.79 \%$ & $0.45 \%$
\end{tabular}




\begin{tabular}{ccccc} 
Adjective & $5.09 \%$ & $2.24 \%$ & $3.21 \%$ & $0.30 \%$ \\
Other & $0.61 \%$ & $0.37 \%$ & $0.32 \%$ & $0.25 \%$ \\
\hline
\end{tabular}

\section{Quantifying feature distinctiveness}

To quantify the feature distinctiveness of words, the feature production data were first transformed into a 4,436 (cues) x 3,981 (features) matrix where each cell represented the proportion of participants who listed a particular feature for a particular cue word. Therefore, each row in this cue-feature matrix represents the feature set distribution of a given word, which is essentially a vector that represents the proportions of participants reporting each feature.

A node was created for each of the 4,436 words in the feature production norms, and each connection represented the distance between two nodes (words) in the feature network. Distance between words was calculated using two different approaches, which make different underlying assumptions about the features involved in the computation. The first approach is Jaccard distance, $d_{j}$, which computes the distance between two words by subtracting the intersection of the two words' feature set distributions (i.e., a vector that represents the proportions of participants reporting each feature for a given word) over their union from 1. In layperson terms, Jaccard distance is sensitive to the extent to which features are shared between the two words. The second approach is Manhattan distance, $d_{m}$, which computes the distance between two words as the sum of absolute differences between the proportions of participants reporting each feature. In layperson terms, Manhattan distance is sensitive to the extent to which features differ between the two words. Both approaches were previously investigated by Engelthaler and Hills (2017) in the context of word learning, and emphasize different aspects of the feature set distributions of words. Given the way that Manhattan and Jaccard distances are computed among words' feature set distributions, it is not surprising that the two variables are moderately negatively correlated, $r$ 
$=-0.39 ; p<.001$. However, the correlation is not a perfect one because each distance measure is ultimately computed on the same information available, and each distance measure weights different aspects of a given word's feature information differently. Specifically, Manhattan distance puts more weight on feature information that distinguishes between the two vectors, whereas Jaccard distance puts more weight on feature information that is shared or common to two vectors. In the present context, Manhattan and Jaccard feature distinctiveness indicates that a word can be distinctive on the basis of somewhat different information. Greater Manhattan feature distinctiveness indicates that a word is distinctive because its features are dissimilar from many other words whereas greater Jaccard feature distinctiveness indicates that a word is distinctive because it does not share as many features with other words.

Finally, the feature distinctiveness of a given word relative to other words in the network (subsequently simply referred to as feature distinctiveness) was defined as the sum of the distances between that word and all other words in the network (i.e., sum of the edges of a node). Formal mathematical descriptions of the two distance measures and an example can be found in Appendix B.

\section{Megastudy data and linguistic norms}

The following sets of behavioral and linguistic data were obtained for the present study: reaction time and item accuracy for visual lexical decision from the English Lexicon Project (Balota et al., 2007), reaction time and item accuracy from the Calgary Semantic Decision Task (Pexman et al., 2017), as well as relevant linguistic norms (age-of-acquisition, concreteness, frequency) for the stimuli.

The English Lexicon Project (ELP) is a large database containing descriptive and behavioral data for over 40,000 English words administered in a visual lexical decision task (see 
Balota et al., 2007, for a complete description of the database). The data can be freely downloaded at http://elexicon.wustl.edu/. In lexical decision, participants indicated if the letter string shown on a computer screen formed a real English word or a nonword by pressing the corresponding buttons on a button box. ELP behavioral data for the visual lexical decision task exist for 4,033 of the 4,436 words $(\sim 91 \%)$ in the feature production norms.

The Calgary Semantic Decision Project (CSDP) is a large database containing behavioral data for 10,000 English words administered in a semantic decision task (see Pexman et al., 2017, for a complete description of the database). In semantic decision, participants indicated if the word was a concrete (e.g., dog, night) or abstract (e.g., beauty, freedom) word by pressing the corresponding buttons on a button box. Behavioral data exist for 1,344 of the 4,436 words $(\sim 30 \%)$ in the feature production norms. As trial-level data was available for the CSDP, these were used to conduct linear mixed-effect modeling in the data analysis.

Finally, additional lexical properties of words analyzed in this paper were obtained from existing lexical norms. Age-of-acquisition ratings were obtained from the Kuperman, Stadthagen-Gonzalez, and Brysbaert (2012) norms, who collected age-of-acquisition ratings for over 30,000 English words. Concreteness ratings were obtained from the Brysbaert, Warriner, and Kuperman (2014) norms, who collected concreteness ratings of 40,000 English words. Additional measures of word frequency, specifically SUBTLEX $X_{U S}$ frequencies obtained from subtitles of television shows and films (Brysbaert \& New, 2009), and orthographic neighborhood size (i.e., the number of words that were orthographically similar to a given word), were obtained from the ELP.

\section{Results}


A series of linear regression analyses were conducted to find out if two measures of feature distinctiveness (i) were associated with earlier age of acquisition ratings, (ii) influenced lexical processing in a lexical decision task, and (iii) influenced semantic processing in a semantic decision task. Recall that the two measures of feature distinctiveness were obtained by computing the Jaccard and Manhattan distances of a particular word's feature set distribution to the feature set distributions of all other words in the database, which represent an explicit consideration of the structure of the "feature space" that words reside in. Hence, a word's feature distinctiveness indicates the extent to which a word's elicited features are distinct, or dissimilar, from those of other words.

In addition, a number of covariates such as word length, word frequency, orthographic neighborhood size, concreteness, word class, age of acquisition, number of (unique) features (i.e., the list of features associated with a given word), were included where appropriate to provide a conservative assessment of whether feature distinctiveness effects accounted for additional variance on top of these covariates. In the analyses below, predictors were mean centered and standardized, and models did not suffer from high levels of multicollinearity (i.e., all variance inflation factors $\sim 4)$.

\section{Data Analysis Plan}

The following data analysis plan was generally adopted across all three sets of analyses in order to explicitly evaluate if two measures of feature distinctiveness significantly contributed to the model in accounting for age of acquisition ratings and behavioral performance on visual lexical decision and semantic decision after considering the influence of covariates. Covariates are control variables that have been previously shown to influence behavioral outcomes in lexical and semantic processing tasks (i.e., length or number of letters, frequency, age of 
acquisition, word class, concreteness, orthographic neighborhood size, number of features), whereas the theoretical variables of interest in the present paper were Manhattan and Jaccard feature distinctiveness. In the first step, covariates were included in the model and submitted to a stepwise algorithm that attempts to find the set of predictors that minimizes AIC of the model. In the second step, theoretical variables of interest were added to the regression model in the first step and submitted to the same stepwise algorithm procedure. A likelihood ratio test comparing the models from the first and second steps was then conducted to determine if the inclusion of measures of feature distinctiveness significantly improved the model.

Although the gold standard in psycholinguistic research is to conduct linear-mixed effects modeling to account for the variability across participants and items (Baayen, Davidson, \& Bates, 2008), this statistical approach requires trial-level data which was unfortunately not available for the age of acquisition ratings and lexical decision data. Hence, linear regression models were analyzed for the item means for the age of acquisition and lexical decision analyses, whereas linear mixed effects models were analyzed for the semantic decision data.

Finally, all data analysis was conducted in the R programming environment. The step() function was used to conduct the stepwise algorithm that attempted to find the set of predictors that minimizes AIC of the model, and lmerTest package (Kuznetsova, Brockhoff, \& Christensen, 2017) was used for the linear mixed effects modeling. Analysis 1: Age-of-Acquisition Ratings

Linear regression was conducted on 4,013 words for which age of acquisition ratings from Kuperman et al. (2012) were available. The dependent variable was age of acquisition ratings. The predictors consisted of the following: number of letters (length), word frequency, concreteness, word class, number of features, and two measures of feature distinctiveness. 
Appendix A (Table A1) shows the correlations between predictors and outcome variable included in this set of analyses.

In the first step, all covariates (length, word frequency, concreteness, word class, number of features) were included in the regression model and submitted to a stepwise algorithm that attempts to find the set of predictors that minimizes AIC of the model. In the second step, theoretical variables of interest (Manhattan and Jaccard feature distinctiveness) were added to the regression model in the first step and submitted to the same stepwise algorithm procedure. A likelihood ratio test comparing the models from step 1 and step 2 indicated that including measures of feature distinctiveness significantly improved the model, $\chi^{2}(1)=30.425, p<.001$.

Table 2 shows the final regression model. The following variables were retained in the final model: Length, frequency, word class, concreteness, number-of-features, and Manhattan feature distinctiveness. Jaccard feature distinctiveness was not retained in the final model. Words of shorter length, higher frequency, higher in concreteness, and that had many features were acquired earlier. In particular, Manhattan feature distinctiveness was a significant predictor of age of acquisition, standardized $\beta=-0.12, t(4003)=-5.52, p<.001$, such that words with greater feature distinctiveness (based on Manhattan distance) were acquired earlier in life. For each standardized unit increase in feature distinctiveness, the average decrease in age of acquisition was 0.12 . Together, the predictors explained $52.7 \%$ of the variance in age of acquisition ratings, accounting for a significant proportion of the variance, $R^{2}=.527, F(9,4003)$ $=495.2, p<.001$.

Table 2. Final regression model for age-of-acquisition ratings.

\begin{tabular}{lcccc}
\hline Predictors & std. Beta & std. Cl & $t$ & $p$ \\
\hline (Intercept) & & & 232.59 & $<.001$
\end{tabular}


FEATURE DISTINCTIVENESS 16

\begin{tabular}{lcccc} 
Length & 0.08 & $0.06-0.11$ & 6.65 & $<.001$ \\
Frequency & -0.57 & $-0.59--0.54$ & -45.09 & $<.001$ \\
POS [adjective] & -0.12 & $-0.14--0.09$ & -9.36 & $<.001$ \\
POS [other] & -0.04 & $-0.07--0.02$ & -3.91 & $<.001$ \\
POS [adverb] & -0.03 & $-0.05--0.01$ & -2.73 & .006 \\
POS [verb] & -0.07 & $-0.09--0.05$ & -5.99 & $<.001$ \\
Concreteness & -0.37 & $-0.40--0.34$ & -25.45 & $<.001$ \\
Number-of-features & -0.06 & $-0.10--0.02$ & -2.85 & .004 \\
Manhattan distinctiveness & -0.12 & $-0.16--0.08$ & -5.52 & $<.001$ \\
\hline Observations & 4013 & & & \\
$\mathrm{R}^{2} /$ adjusted $\mathrm{R}^{2}$ & $0.527 / 0.526$ & & & \\
\hline
\end{tabular}

Legend: POS = part of speech/word class [baseline: nouns]

\section{Analysis 2: English Lexicon Project: Lexical Decision Task}

Item-level regression analyses were conducted on the mean RTs and accuracies for 4,013 words for the visual lexical decision task obtained from the ELP. The dependent variables consisted of $z$-scored RTs and accuracy rates, averaged across participants for each word. Each participant's raw lexical decision latencies were first standardized via a $z$-score transformation, and the mean $z$-score for all participants presented with a particular item was then computed for that word (Balota et al., 2007). Although both raw and $z$-scored RTs are available in the ELP, $z$ scored RTs, instead of raw RTs, were analyzed to reduce the likelihood that any single participant may disproportionately influence the item means.

The predictors consisted of the following: number of letters (length), word frequency, age of acquisition, orthographic neighborhood size, concreteness, word class, number of features, and two measures of feature distinctiveness. Appendix A (Table A2) shows the correlations between predictors and outcome variables included in this set of analyses.

In the first step, all covariates (length, word frequency, age of acquisition, concreteness, word class, number of features) were included in the regression model and submitted to a 
stepwise algorithm that attempts to find the set of predictors that minimizes AIC of the model. In the second step, theoretical variables of interest (Manhattan and Jaccard feature distinctiveness) were added to the regression model in the first step and submitted to the same stepwise algorithm procedure. A likelihood ratio test comparing the models from step 1 and step 2 indicated that including measures of feature distinctiveness significantly improved the RT model: $\chi^{2}(1)=$ $30.425, p<.001$. For the Accuracy model, the stepwise procedure in step 2 found the same model as in step 1, indicating that including measuring of feature distinctiveness did not significantly improve the model.

Table 3 shows the final regression models on $z$-scored lexical decision RTs and item accuracies. For the RT model, the following variables were retained: Length, frequency, age of acquisition, orthographic neighborhood size, concreteness, word class, number of features, and Manhattan feature distinctiveness. Jaccard feature distinctiveness was not retained in the final model. Words of shorter length, higher frequency, lower age of acquisition ratings, with more orthographic neighbors, lower in concreteness, and that had many features were responded to more quickly in visual lexical decision. In particular, Manhattan feature distinctiveness was a significant predictor of RTs, standardized $\beta=0.07, t(4013)=3.43, p=.001$, such that words with greater feature distinctiveness were more slowly responded to in lexical decision. For each standardized unit increase in feature distinctiveness, the average increase in $z$-scored RT was 0.07. Together, the predictors explained $54.7 \%$ of the variance in lexical decision RTs, accounting for a significant proportion of the variance in lexical decision $\mathrm{RTs}, R^{2}=.547, F(11$, 4001) $=439.7, p<.001$.

For the Accuracy model, the following variables were retained: Length, frequency, and age of acquisition, concreteness, word class. Orthographic neighborhood size, number of 
features, Manhattan and Jaccard feature distinctiveness were not retained in the final model.

Words that were longer, of higher frequency, lower age of acquisition, and lower concreteness were responded to more accurately in visual lexical decision. Together, the predictors explained $20.6 \%$ of the variance in lexical decision accuracies, accounting for a significant proportion of the variance in lexical decision accuracies, $R^{2}=.195, F(8,4004)=130, p<.001$. 
Table 3. Final regression models for visual lexical decision (RTs and Accuracy).

\begin{tabular}{|c|c|c|c|c|c|c|c|c|}
\hline \multirow[b]{2}{*}{ Predictors } & \multicolumn{4}{|c|}{ RT } & \multicolumn{4}{|c|}{ Accuracy } \\
\hline & std. $\beta$ & std. Cl & $t$ & $p$ & std. $\beta$ & std. Cl & $t$ & $p$ \\
\hline (Intercept) & & & -129.71 & $<.001$ & & & 795.58 & $<.001$ \\
\hline Length & 0.35 & $0.32-0.38$ & 23.63 & $<.001$ & 0.19 & $0.16-0.22$ & 11.77 & $<.001$ \\
\hline Frequency & -0.45 & $-0.48--0.42$ & -29.84 & $<.001$ & 0.37 & $0.34-0.41$ & 18.85 & $<.001$ \\
\hline Age of acquisition & 0.21 & $0.18-0.24$ & 13.45 & $<.001$ & -0.20 & $-0.24--0.16$ & -9.95 & $<.001$ \\
\hline $\begin{array}{l}\text { Orthographic } \\
\text { neighborhood }\end{array}$ & 0.07 & $0.04-0.10$ & 4.85 & $<.001$ & & & & \\
\hline Concreteness & 0.04 & $0.01-0.07$ & 2.70 & .007 & -0.06 & $-0.10--0.03$ & -3.35 & .001 \\
\hline POS [adjective] & 0.02 & $0.00-0.05$ & 2.01 & .045 & -0.02 & $-0.05-0.01$ & -1.35 & .178 \\
\hline POS [other] & 0.11 & $0.09-0.14$ & 10.1 & $<.001$ & -0.10 & $-0.13--0.07$ & -6.65 & $<.001$ \\
\hline POS [adverb] & 0.06 & $0.04-0.08$ & 5.66 & $<.001$ & -0.06 & $-0.09--0.03$ & -4.06 & $<.001$ \\
\hline POS [verb] & 0.05 & $0.02-0.07$ & 3.96 & $<.001$ & -0.03 & $-0.06-0.00$ & -1.83 & .068 \\
\hline Number-of-features & -0.05 & $-0.09--0.01$ & -2.42 & .016 & & & & \\
\hline Manhattan distinctiveness & 0.07 & $0.03-0.11$ & 3.43 & .001 & & & & \\
\hline Observations & 4013 & & & & 4013 & & & \\
\hline $\mathrm{R}^{2} /$ adjusted $\mathrm{R}^{2}$ & $0.547 / 0.546$ & & & & $0.206 / 0.205$ & & & \\
\hline
\end{tabular}

Legend: $\mathrm{POS}=$ part of speech/word class [baseline: nouns] 


\section{Analysis 3: Calgary Semantic Decision Project: Semantic Decision Task}

Linear mixed effects analysis was conducted on the trial-level RTs for 36,815 observations for the semantic decision task obtained from the Calgary SDP and item-level regression analysis was conducted on the mean accuracies for 1,336 words for the semantic decision task obtained from the Calgary $\mathrm{SDP}^{1}$. The dependent variable for the linear mixed effects model was $z$-scored RTs and the dependent variable for the item-level regression model was accuracy rates averaged across participants for each word. As described previously, $z$-scored RTs, instead of raw RTs, were analyzed to reduce the likelihood that any single participant may disproportionately influence the item means.

The predictors consisted of the following: number of letters (length), word frequency, age of acquisition, concreteness, orthographic neighborhood size, word class, number of features, and two measures of feature distinctiveness. In addition, two interaction terms (Manhattan distinctiveness $\times$ Concreteness, Jaccard distinctiveness $\times$ Concreteness) were included as predictors to specifically assess if feature distinctiveness effects could be generalized across words with varying levels of concreteness ${ }^{2}$. Appendix A (Table A3) shows the correlations between predictors and outcome variables included in this set of analyses.

For the linear mixed-effects analysis of semantic decision RTs, the random part of the model was first modeled. The by-participant or by-item random intercepts were tested to determine if they should be included in the model. Likelihood ratio tests indicated that the random effects of participants, $\chi^{2}(1)=50.36, p<.001$, and the random effects of items, $\chi^{2}(1)=$ $3525.5, p<.001$, should be included in the model. With the random effects structure determined for the RT model, model building and testing proceeded in the same way for both RT and Accuracy models. 
In the first step, all covariates (length, word frequency, age of acquisition, concreteness, word class, number of features) were included in the regression model and submitted to a stepwise algorithm that attempts to find the set of predictors that minimizes AIC of the model. In the second step, theoretical variables of interest (Manhattan and Jaccard feature distinctiveness) were added to the regression model in the first step and submitted to the same stepwise algorithm procedure. In the third step, two interaction terms (Manhattan distinctiveness $\times$ Concreteness, Jaccard distinctiveness $\times$ Concreteness) were added to the regression model in the second step and submitted to the same stepwise algorithm procedure.

A likelihood ratio test comparing the models from step 1 and step 2 indicated that including measures of feature distinctiveness significantly improved the RT model: $\chi^{2}(1)=$ 11.29, $p<.001$, and the Accuracy model: $\chi^{2}(2)=14.71, p<.001$. A likelihood ratio test comparing the models from step 2 and step 3 indicated that including the interaction terms between feature distinctiveness measures and concreteness significantly improved the RT model: $\chi^{2}(3)=24.50, p<.001$, and the Accuracy model: $\chi^{2}(2)=48.99, p<.001$.

Table 4 shows the final linear mixed effects and linear regression models for $z$-scored semantic decision RTs and accuracies. Note that both interaction effects (Manhattan distinctiveness $\times$ Concreteness, Jaccard distinctiveness $\times$ Concreteness) were retained in both the RT and Accuracy models.

For the RT model, the following variables were retained: Length, frequency, age of acquisition, orthographic neighborhood size, concreteness, word class, Manhattan and Jaccard feature distinctiveness, interaction between concreteness and Manhattan feature distinctiveness, and interaction between concreteness and Jaccard feature distinctiveness. Number of features was not retained in the final model. Words with fewer letters, of higher frequency, of earlier age 
of acquisition, with fewer orthographic neighbors, and higher concreteness were more quickly responded to in the semantic decision task. Although the main effects of Manhattan and Jaccard feature distinctiveness were not significant predictors, this was qualified by significant interactions with concreteness: Manhattan distinctiveness $\times$ Concreteness, standardized $\beta=$ $0.08, t(1260.8)=-4.88, p<.001 ;$ Jaccard distinctiveness $\times$ Concreteness, standardized $\beta=0.03$, $t(1259.3)=3.06, p=.002$. Together, the predictors explained $8.5 \%$ of the variance in semantic decision RTs.

For the Accuracy model, the following variables were retained: Length, frequency, age of acquisition, orthographic neighborhood size, concreteness, word class, Manhattan and Jaccard feature distinctiveness, interaction between concreteness and Manhattan feature distinctiveness, and interaction between concreteness and Jaccard feature distinctiveness. Number of features was not retained in the final model. Words of earlier age of acquisition, with fewer orthographic neighbors, higher concreteness, greater Jaccard feature distinctiveness were more accurately responded to in the semantic decision task. Although the main effect of Manhattan feature distinctiveness was not a significant predictor, this and the significant main effect of Jaccard feature distinctiveness was qualified by significant interactions with concreteness: Manhattan distinctiveness $\times$ Concreteness, standardized $\beta=0.21, t(1323)=4.38, p<.001 ;$ Jaccard distinctiveness $\times$ Concreteness, standardized $\beta=-0.11, t(1323)=-3.95, p<.001$. Together, the predictors explained $16.2 \%$ of the variance in semantic decision accuracies, accounting for a significant proportion of the variance in semantic decision accuracies, $R^{2}=.162, F(12,1323)=$ 21.32, $p<.001$.

As there were significant interaction effects in the final models, additional simple slope analyses were conducted to clearly understand the nature of the interaction between measures of 
feature distinctiveness and concreteness. Specifically, the slope/effect of feature distinctiveness was evaluated at two levels of concreteness ( $+1 S D$ above the mean and $-1 S D$ below the mean) to determine if feature distinctiveness effects were similar for concrete and abstract concepts. Table 5 shows the results of the simple slopes analyses. Figures 1 and 2 shows a graphical depiction of the interactions. Overall, the effect of feature distinctiveness differed across concrete and abstract concepts. For concrete words, there was a significant Manhattan feature distinctiveness effect, such that concrete words with higher Manhattan feature distinctiveness were more quickly and accurately responded to in semantic decision. For abstract words, the Manhattan feature distinctiveness effect was reversed, such that abstract words with lower Manhattan feature distinctiveness were more quickly and accurately responded to in semantic decision. The effect of Jaccard feature distinctiveness was less clear and less robust than that of Manhattan feature distinctiveness; although the simple slopes analyses suggested that concrete words with greater Jaccard feature distinctiveness were more slowly responded to, and that abstract words with greater Jaccard feature distinctiveness were more accurately responded to. Together, the pattern of simple slopes indicate a complex pattern of feature distinctiveness effect modulated by concreteness: Concrete words experienced the greatest processing advantage when they were high on Manhattan feature distinctiveness but low on Jaccard feature distinctiveness, whereas abstract words experienced the greatest processing advantage when they were low on Manhattan feature distinctiveness, but high on Jaccard feature distinctiveness. 
FEATURE DISTINCTIVENESS 24

Table 4. Regression results for semantic decision (RTs and Accuracy).

\begin{tabular}{|c|c|c|c|c|c|c|c|c|}
\hline \multirow[b]{2}{*}{ Predictors } & \multicolumn{4}{|c|}{ RT } & \multicolumn{4}{|c|}{ Accuracy } \\
\hline & std. $\beta$ & std. $\mathrm{Cl}$ & $t$ & $p$ & std. $\beta$ & std. $\mathrm{Cl}$ & $t$ & $p$ \\
\hline (Intercept) & & & -16.62 & $<.001$ & & & 170.75 & $<.001$ \\
\hline Length & 0.03 & $0.00-0.05$ & 2.35 & .019 & 0.05 & $-0.01-0.12$ & 1.66 & .097 \\
\hline Frequency & -0.07 & $-0.09--0.05$ & -6.40 & $<.001$ & 0.06 & $-0.01-0.12$ & 1.69 & .091 \\
\hline Age of acquisition & 0.11 & $0.09-0.13$ & 8.98 & $<.001$ & -0.16 & $-0.23--0.10$ & -4.64 & $<.001$ \\
\hline Orthographic neighborhood & 0.03 & $0.01-0.05$ & 2.64 & .008 & -0.09 & $-0.15--0.02$ & -2.70 & .007 \\
\hline POS [adjective] & 0.00 & $-0.02-0.02$ & -0.30 & .761 & 0.05 & $-0.01-0.11$ & 1.78 & .075 \\
\hline POS [adverb] & 0.00 & $-0.02-0.02$ & 0.13 & .897 & 0.03 & $-0.02-0.08$ & 1.00 & .319 \\
\hline POS [verb] & 0.03 & $0.01-0.05$ & 3.27 & .001 & -0.05 & $-0.11-0.00$ & -1.88 & .06 \\
\hline Concreteness & -0.21 & $-0.24--0.17$ & -12.61 & $<.001$ & 0.31 & $0.22-0.40$ & 6.69 & $<.001$ \\
\hline Manhattan distinctiveness & 0.02 & $-0.02-0.06$ & 1.20 & .232 & -0.10 & $-0.20-0.01$ & -1.73 & .083 \\
\hline \multirow[t]{2}{*}{ Jaccard distinctiveness } & 0.01 & $-0.01-0.03$ & 0.89 & .372 & 0.06 & $0.01-0.12$ & 2.22 & .027 \\
\hline & \multicolumn{4}{|c|}{ Interaction Effects } & \multicolumn{4}{|c|}{ Interaction Effects } \\
\hline Manhattan $\times$ Concreteness & -0.08 & $-0.12--0.05$ & -4.88 & $<.001$ & 0.21 & $0.12-0.31$ & 4.38 & $<.001$ \\
\hline \multirow[t]{2}{*}{ Jaccard × Concreteness } & 0.03 & $0.01-0.05$ & 3.06 & .002 & \multirow[t]{5}{*}{-0.11} & \multirow[t]{5}{*}{$-0.16--0.06$} & \multirow[t]{5}{*}{-3.95} & \multirow[t]{5}{*}{$<.001$} \\
\hline & \multicolumn{4}{|c|}{ Random Effects } & & & & \\
\hline$\sigma^{2}$ & 0.66 & & & & & & & \\
\hline$\tau_{00}$ & 0.05 cue & 0.00 Participant & & & & & & \\
\hline ICC & 0.07 cue & 0.01 Participant & & & & & & \\
\hline Observations & 36815 & & & & 1336 & & & \\
\hline Marginal $\mathrm{R}^{2} /$ Conditional $\mathrm{R}^{2}$ & $0.085 / 0.159$ & & & & $0.162 / 0.154$ & & & \\
\hline
\end{tabular}

Legend: $P O S=$ part of speech/word class [baseline: nouns] 
Table 5. Simple slope analysis of significant interaction effects in the semantic decision task.

\begin{tabular}{|c|c|c|c|c|c|c|c|c|}
\hline \multicolumn{5}{|c|}{ RT } & \multicolumn{4}{|c|}{ ACC } \\
\hline \multicolumn{9}{|c|}{ Simple effect of Manhattan feature distinctiveness } \\
\hline Concreteness & Estimate & $S E$ & $d f$ & $p$ & Estimate & $S E$ & $d f$ & $p$ \\
\hline +1 SD above mean & -0.07 & 0.01 & -6.44 & $<.001$ & 0.02 & 0.00 & 4.46 & $<.001$ \\
\hline-1 SD below mean & 0.10 & 0.03 & 3.18 & $<.001$ & -0.04 & 0.01 & -3.22 & $<.001$ \\
\hline \multicolumn{9}{|c|}{ Simple effect of Jaccard feature distinctiveness } \\
\hline Concreteness & Estimate & $S E$ & $d f$ & $p$ & Estimate & $S E$ & $d f$ & $p$ \\
\hline+1 SD above mean & 0.04 & 0.01 & 3.05 & $<.001$ & -0.01 & 0.01 & -1.42 & .16 \\
\hline-1 SD below mean & -0.02 & 0.01 & -1.47 & .14 & 0.02 & 0.01 & 4.18 & $<.001$ \\
\hline
\end{tabular}


Figure 1. Interaction plots for RTs in the semantic decision task (left: Manhattan feature distinctiveness; right: Jaccard feature

distinctiveness). Solid lines indicate the effect of feature distinctiveness for abstract concepts; dashed lines indicate the effect of feature distinctiveness for concrete concepts.
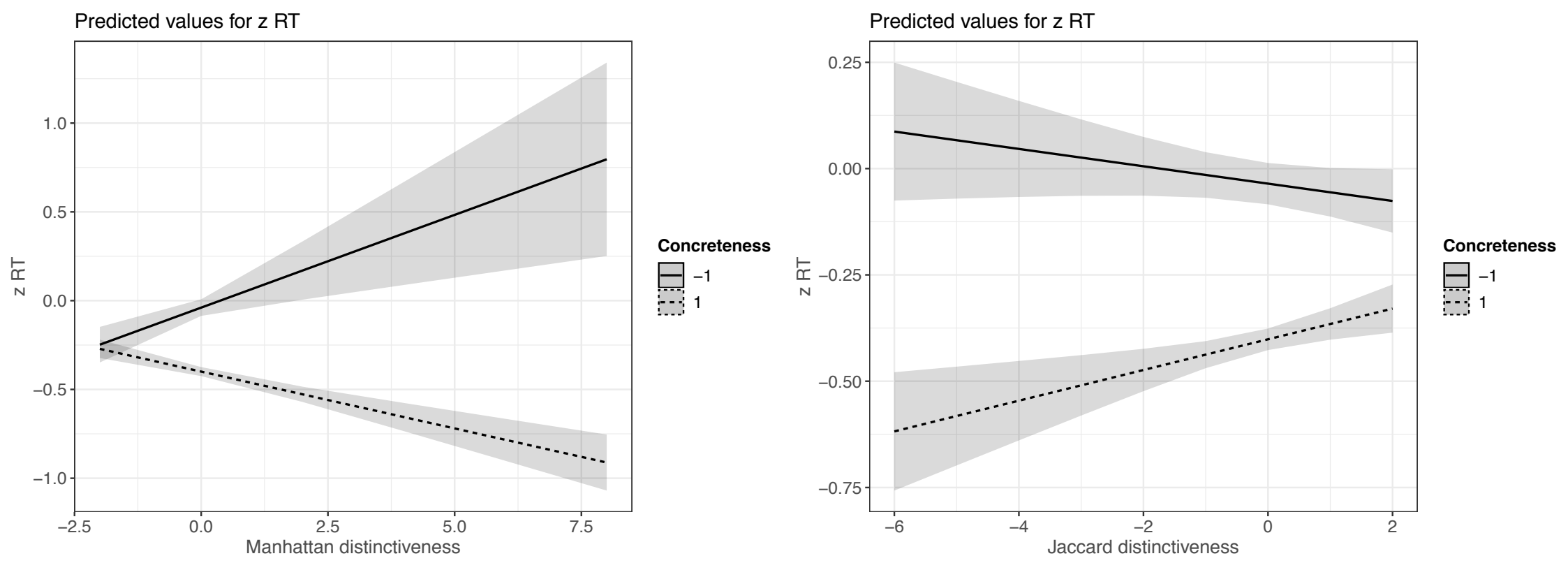
Figure 2. Interaction plots for ACCs in the semantic decision task (left: Manhattan feature distinctiveness; right: Jaccard feature distinctiveness). Solid lines indicate the effect of feature distinctiveness for abstract concepts; dashed lines indicate the effect of feature distinctiveness for concrete concepts.
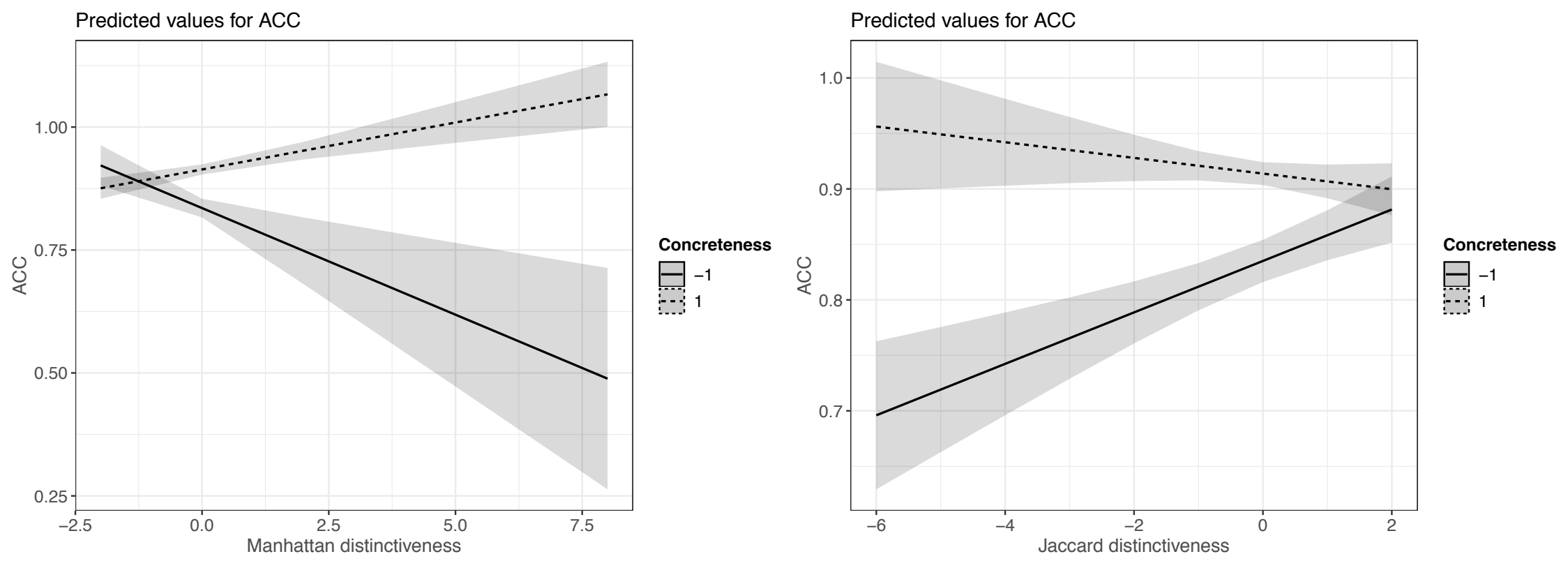
FEATURE DISTINCTIVENESS 28

\section{General Discussion}

The analyses conducted above yielded three main findings. First, Manhattan feature distinctiveness, which emphasizes the influence of distinctive features, was a stronger predictor of age of acquisition ratings and the behavioral data as compared to Jaccard feature distinctiveness, which emphasizes the influence of shared features. Second, words with greater feature distinctiveness tended to be acquired earlier in life. Finally, the nature of feature distinctiveness effects on lexical processing appeared to be task-dependent, where greater Manhattan feature distinctiveness facilitated semantic decision (for concrete concepts and not for abstract concepts) but inhibited lexical decision.

\section{Distinctive versus shared features}

Recall that two different approaches were taken to compute the distance between words in the feature network: Jaccard distance and Manhattan distance. Each approach computes distance somewhat differently_-Jaccard distance emphasized shared features, whereas Manhattan distance emphasized features that are different or dissimilar. In the analyses above, feature distinctiveness computed using Manhattan distances was more predictive of age-ofacquisition ratings and behavioral responses in lexical and semantic decision tasks, as compared to feature distinctiveness computed using Jaccard distances as Jaccard feature distinctiveness was not retained by the stepwise algorithm in the final regression models for the age of acquisition and lexical decision analyses. This finding is in line with Engelthaler and Hills (2017)'s observation that Manhattan distance outperformed other distance measures (including Jaccard distance), and further suggests that people might have greater sensitivity to features that make a concept distinctive and different from other concepts, rather than features that are common to various sets of concepts. This also highlights the fact that while there are many ways 
(e.g., cosine, Euclidean, and many others) of measuring "distance" between lexical items that have been represented as mathematical objects (i.e., a vector of numbers), it is important to carefully consider the kinds of information that each distance measure is capturing and apply them judicially to address our research questions.

Early acquired words tend to have more distinctive features

The next key finding was that feature distinctiveness was associated with earlier age-ofacquisition, such that distinctive words are more likely to be acquired earlier in life. This replicates Engelthaler and Hills (2017)'s original finding with a much larger database of feature production norms - over 4,000 words in the present analysis compared to the 541 words in the McRae et al. (2005) feature norms analyzed by Engelthaler and Hills. Furthermore, this relationship held even after the effects of length, frequency, concreteness, word class, and number of features were accounted for in the regression analysis.

Engelthaler and Hills argued that distinctive objects tend to be acquired early in life because highly dissimilar objects are less likely to be assigned to the same label as compared to similar objects. Nevertheless, much remains to be done to fully understand the mechanisms and processes underlying the finding that distinctive words have a learning advantage, even into early adulthood. For example, are there particular groups or categories of features that are most beneficial for driving the acquisition of new words? Would different sets of features drive the acquisition of concrete or abstract concepts? One way of addressing these intriguing questions is to apply supervised machine learning approaches to find clusters of features that are most predictive of age-of-acquisition ratings. This could be a promising approach to discover distributions of semantic features that are especially salient to children (and adults) as they are 
acquiring a vocabulary of concepts and generalizing these concepts to various categories (Love, Medin, \& Gureckis, 2004; Sloutsky \& Fisher, 2004).

Feature distinctiveness effects in processing are modulated by task demands

The present paper investigated feature distinctiveness effects in lexical decision and semantic decision, using behavioral data obtained from megastudies. This section focusses on how the effects of Manhattan feature distinctiveness differ across the visual lexical decision and semantic decision tasks. Manhattan feature distinctiveness was a significant predictor of behavioral performance on both the visual lexical decision task and the semantic decision task. The effects of Manhattan feature distinctiveness were significant, even after covariates such as frequency, length, age of acquisition, orthographic neighborhood size, concreteness, word class, and number of features were taken into account in the regression analyses.

In lexical decision, words with greater Manhattan feature distinctiveness were more slowly recognized, whereas in semantic decision, the pattern of finding was more complexspecifically concrete words with greater Manhattan feature distinctiveness were more quickly and more accurately responded to whereas abstract words with greater Manhattan feature distinctiveness were more slowly and less accurately responded to. It appears that in a straightforward word recognition task (i.e., decide if a string of letters formed a real word or not), having distinctive features impedes lexical decision performance. On the other hand, in semantic decision where participants had to decide whether words were concrete or abstract concepts, there was a processing advantage associated for concrete words with distinctive features and for abstract words with less distinctive features.

One speculative account of this particular pattern of findings involves considering how spreading activation processes might operate within the semantic structure of language (Collins 
$\&$ Loftus, 1975). If the activation of a lexical node can activate the features that are associated with that particular word (Pexman, Lupker, \& Hino, 2002; Rodd, Gaskell, \& Marslen-Wilson, 2002), we could assume that activation continues to spread in this lexical network such that words that share similar features with the initially activated word become activated themselves (for an example of how such an account might be formally implemented, see Siew, 2019). When a word with low distinctiveness is activated, this would tend to activate many other words in the network because these words share many similar features (low feature distance) as compared to the case when a word with high distinctiveness is activated, which would tend to activate few words because few words share the same features (high feature distance).

Now consider the nature of the two tasks. In the case of lexical decision, words with low feature distinctiveness may lead to the activation of many lexical nodes. This widespread activation within the system might lead to rapid accumulation of evidence that, within the context of diffusion models in lexical decision (e.g., Ratcliff, Gomez, \& McKoon, 2004), would allow for a steeper drift rate that facilitates a faster WORD decision to the stimulus. On the other hand, in a task that emphasizes accessing the semantic properties of a word, words with high feature distinctiveness may lead to the activation of $f e w$ lexical nodes, which could facilitate processing because there is less competition for the word on which a semantic decision must be made. This allows its semantic representation to emerge more robustly and to be more distinguishable from other words (Rodd et al., 2002), and making it easier to make a concrete versus abstract judgment on such words. Recall that the Manhattan feature distinctiveness advantage was observed for concrete words in semantic decision, but the opposite pattern was observed for abstract words where lower Manhattan feature distinctiveness was associated with worse performance on the task. Hence, this admittedly speculative account might be only 
applicable to concrete concepts. Processing of abstract words might be driven by a different mechanism where diffuse but widespread activation of many nodes in the semantic space provides strong evidence for an "abstract" decision to be made—not an entirely implausible account given recent proposals that suggest that the representation of abstract words is likely multidimensional and involves multiple systems, including linguistic, perceptual, sensorimotor, and valence systems, that are invoked to varying extents in the computation or simulation of meaning (Louwerse \& Jeuniaux, 2010; Zdrazilova \& Pexman, 2013).

It certainly remains for future work to provide a computational account of the speculative verbal explanations of feature distinctiveness effects described above, and uncover the mechanisms that drive these effects in the processing of concrete and abstract concepts. Additional investigations that examines how this new measure of feature distinctiveness interacts with other variables of semantic richness, such as semantic neighbors and contextual diversity (Pexman et al., 2008; Yap et al., 2011) are needed to gain new insights into semantic processing. Feature distinctiveness effects in semantic processing are modulated by word concreteness

In this section, we turn to the intriguing interaction effects between feature distinctiveness and concreteness in the semantic decision analyses. The nature of these interaction effects is quite complex. The simple slope analysis indicates that the effects of Manhattan and Jaccard feature distinctiveness differ for concrete and abstract words. For concrete words, greater Manhattan feature distinctiveness facilitated semantic processing (faster RTs and higher ACCs) but greater Jaccard feature distinctiveness inhibited semantic processing (faster RTs only). For abstract words, greater Manhattan feature distinctiveness inhibited semantic processing (slower RTs and lower ACCs) but greater Jaccard feature distinctiveness facilitated semantic processing (higher ACCs only). 
Recall that Jaccard distance is sensitive to the extent to which features are shared between the two words whereas Manhattan distance is sensitive to the extent to which features differ between the two words. The facilitatory effect of Manhattan feature distinctiveness and inhibitory effect of Jaccard feature distinctiveness for concrete words suggest that, during the processing of concrete concepts, possessing feature information that distinguishes or discriminates between similar concepts (i.e., Manhattan distance) as well as feature information that is shared among many other concepts (i.e., Jaccard distance) are both important factors in semantic computation. This is consistent with much previous word meaning research, where both distinctive and shared features are important for identifying concepts (Cree et al., 2006; Montefinese, Vinson, \& Ambrosini, 2018; Montefinese, Zannino, \& Ambrosini, 2015; Taylor, Devereux, Acres, Randall, \& Tyler, 2012). On the other hand, possessing feature information that does not distinguish or discriminate between similar concepts (i.e., Manhattan distance) as well as feature information that is not shared among many other concepts (i.e., Jaccard distance) appear to be important in the semantic processing of abstract concepts. However, it is important to interpret this pattern of results within the context of task demands in semantic decision where a forced binary decision of concrete vs. abstract was made in response to a given concept. It is possible that semantic processes prioritized detection of concrete concepts and the accumulation of positive evidence (i.e., having discriminating features or commonly shared features) for a "concrete" decision, and less so for "abstract" decisions.

\section{Implications for models of semantic memory}

As discussed in the Introduction, this paper attempts to bring together two competing theoretical approaches in semantic memory research — the meaning of a word (i) can be decomposed into smaller units (i.e., decompositional theories; Rips et al, 1973) and (ii) depends 
on its position in a broader semantic network or space (i.e., semantic network model; Collins \& Loftus, 1975). In this paper the internal properties of words (i.e., their semantic features from feature production norms) were used determine where words are located in an external, semantic feature space (i.e., whether a word was distinctive relative to other words), and analyses of megastudy data showed that a word's distinctiveness in the global semantic feature space affect performance in semantic processing. Here the implications of these findings for decompositional (or componential) and network models of semantic memory are briefly discussed.

One prominent framework that emphasizes the importance of the internal structure of concept is the Conceptual Structure Account (CSA), developed primarily to account for patterns of semantic category deficits among patients with acquired neurological impairment (Moss et al., 1997; Moss, Tyler, \& Taylor, 2007; Taylor, Devereux, \& Tyler, 2011). The CSA's main argument is that differential deficits in semantic categories (e.g., living vs. non-living, nouns vs. verbs) can be accounted for by considering how the internal structure of conceptual representations varies across different categories through examining feature intercorrelations of concepts across domains. When considered through the lens of the CSA, the present finding that Manhattan feature distinctiveness of words in a semantic feature network facilitated processing of concrete concepts but not abstract concepts fuels an intriguing hypothesis that the role of distinctive features in semantic computation may vary as function of category (concrete vs. abstract) due to differences in the internal featural composition of concrete and abstract concepts. Indeed, one limitation of the current approach is that the computation of distance measures was somewhat coarse-grained in that all features were treated similarly, even though features represent different knowledge types (e.g., perceptual or functional) and might be more informative for one domain than another (Peters \& Borovsky, 2019). One future direction would 
be to obtain knowledge types for features listed in the Buchanan et al. (2019) feature production norms and re-compute feature distinctiveness measures by weighting features differently in the feature network.

The approach taken in the present paper treats each word as a node in a semantic network and computes semantic distance between all possible pairs of words in the network. This approach is conceptually close to semantic networks of semantic memory (Collins \& Loftus, 1975; Steyvers \& Tenenbaum, 2005). In early models, the distance (i.e., length of the connection) between two nodes in the network was negatively correlated with the number of shared features between those two concepts (Collins \& Loftus, 1975). In more recent network models, nodes were either connected if they shared at least one feature or connections were weighted by the number of shared features (Hills et al., 2009; Peters \& Borovsky, 2019; Sizemore et al., 2018). In contrast, the connections in the present feature distinctiveness network considers all of the information in the feature set distribution vectors associated with each concept to compute distances between pairs of words (i.e., not just considering set overlap/shared features). Taken together, the present paper provides an important demonstration of how the internal feature composition of concepts can be utilized to enhance investigations into the overall structure of an (external) semantic feature space, which has led to some insights into the mechanisms underlying semantic computation and processing.

Finally, it is noteworthy that feature distinctiveness effects were found even though number of features was included as a predictor in the regression models. Number of features was not a significant predictor of age-of-acquisition and SDT performance when feature distinctiveness was included as a predictor. This highlights the importance of going beyond a straightforward count of features associated with a given word, and adopting and developing 
measures that explicitly consider the structural properties of words in a semantic feature space. As demonstrated in many areas of the cognitive sciences and psycholinguistics (see Siew et al., 2019, for an overview), techniques from network analysis can provide us with ways to quantify and measure form-similarity (e.g., Siew \& Vitevitch, 2019) and semantic relationships (e.g., Steyvers \& Tenenbaum, 2005) among thousands of words in the mental lexicon. Quantifying the structural properties of words in a semantic feature space using network analysis can deepen our understanding of the factors that drive language acquisition and lexical processing.

\section{Conclusion}

To recapitulate, the key results from this paper include a replication of Engelthaler and Hills (2017)'s finding that words with greater feature distinctiveness tend to be acquired early in life, the finding that feature distinctiveness effects on lexical processing appeared to be taskdependent, where greater feature distinctiveness facilitated semantic decision but inhibited lexical decision, as well as the finding that the effects of feature distinctiveness on semantic decision differed for concrete and abstract concepts. By combining the availability of lexical and behavioral databases with powerful quantitative tools such as network analysis, this paper demonstrated how we can quantify the structure of feature networks to further our understanding of language acquisition and processing. 
Author's Note

The author thanks Nichol Castro for providing useful comments on an earlier draft of this manuscript.

Data and analysis scripts are available on the Open Science Framework: https://osf.io/X87tr/?view_only=9e4ac81498ef471792cd1cdc349be60f (view-only link for peer review)

\section{Footnotes}

${ }^{1}$ Using a logistic mixed effects model for analyzing accuracy at the trial level led to model convergence issues and/or to degenerate models due to model complexity. Hence, accuracy for the semantic decision task was analyzed at the item-level using linear regression.

${ }^{2}$ It is important to note that this additional analysis of interaction effects was post-hoc in nature and not an a priori research question. The analysis was conducted in response to a reviewer who felt that it was particularly important to test if feature distinctiveness effects were consistent across concrete and abstract concepts.

\section{Compliance with Ethical Standards}

Funding: No funding to declare.

Ethical Approval: This article does not contain any studies with human participants performed by any of the authors.

Conflict of Interest: C. S. Q. S. declares that she has no conflict of interest. 
FEATURE DISTINCTIVENESS 38

\section{References}

Adelman, J. S., Brown, G. D., \& Quesada, J. F. (2006). Contextual diversity, not word frequency, determines word-naming and lexical decision times. Psychological Science, 17(9), 814-823.

Baayen, R. H., Davidson, D. J., \& Bates, D. M. (2008). Mixed-effects modeling with crossed random effects for subjects and items. Journal of Memory and Language, 59(4), 390412.

Balota, D. A., Yap, M. J., Hutchison, K. A., Cortese, M. J., Kessler, B., Loftis, B., ... Treiman, R. (2007). The English lexicon project. Behavior Research Methods, 39(3), 445-459.

Booth, A. E., \& Waxman, S. (2002). Object names and object functions serve as cues to categories for infants. Developmental Psychology, 38(6), 948.

Brysbaert, M., \& New, B. (2009). Moving beyond Kučera and Francis: A critical evaluation of current word frequency norms and the introduction of a new and improved word frequency measure for American English. Behavior Research Methods, 41(4), 977-990.

Brysbaert, M., Warriner, A. B., \& Kuperman, V. (2014). Concreteness ratings for 40 thousand generally known English word lemmas. Behavior Research Methods, 46(3), 904-911. https://doi.org/10.3758/s13428-013-0403-5

Buchanan, E. M., Holmes, J. L., Teasley, M. L., \& Hutchison, K. A. (2013). English semantic word-pair norms and a searchable Web portal for experimental stimulus creation. Behavior Research Methods, 45(3), 746-757. https://doi.org/10.3758/s13428-012-0284-z

Buchanan, E. M., Valentine, K. D., \& Maxwell, N. P. (2019). English semantic feature production norms: An extended database of 4436 concepts. Behavior Research Methods. https://doi.org/10.3758/s13428-019-01243-z 
FEATURE DISTINCTIVENESS 39

Bullinaria, J. A., \& Levy, J. P. (2007). Extracting semantic representations from word cooccurrence statistics: A computational study. Behavior Research Methods, 39(3), 510526.

Castro, N., \& Stella, M. (2019). The multiplex structure of the mental lexicon influences picture naming in people with aphasia. Journal of Complex Networks. https://doi.org/10.1093/comnet/cnz012

Collins, A. M., \& Loftus, E. F. (1975). A spreading-activation theory of semantic processing. Psychological Review, 82(6), 407.

Cree, G. S., McNorgan, C., \& McRae, K. (2006). Distinctive features hold a privileged status in the computation of word meaning: Implications for theories of semantic memory. Journal of Experimental Psychology: Learning, Memory, and Cognition, 32(4), 643.

Cree, G. S., \& McRae, K. (2003). Analyzing the factors underlying the structure and computation of the meaning of chipmunk, cherry, chisel, cheese, and cello (and many other such concrete nouns). Journal of Experimental Psychology: General, 132(2), 163.

De Deyne, S., Navarro, D. J., Perfors, A., Brysbaert, M., \& Storms, G. (2018). The "Small World of Words" English word association norms for over 12,000 cue words. Behavior Research Methods, 1-20.

De Deyne, S., \& Storms, G. (2008). Word associations: Network and semantic properties. Behavior Research Methods, 40(1), 213-231.

Devereux, B. J., Tyler, L. K., Geertzen, J., \& Randall, B. (2014). The Centre for Speech, Language and the Brain (CSLB) concept property norms. Behavior Research Methods, 46(4), 1119-1127. 
Engelthaler, T., \& Hills, T. T. (2017). Feature biases in early word learning: Network distinctiveness predicts age of acquisition. Cognitive Science, 41, 120-140.

Firth, J. R. (1957). A synopsis of linguistic theory, 1930-1955. Studies in Linguistic Analysis.

Garrard, P., Lambon Ralph, M. A., Hodges, J. R., \& Patterson, K. (2001). Prototypicality, distinctiveness, and intercorrelation: Analyses of the semantic attributes of living and nonliving concepts. Cognitive Neuropsychology, 18(2), 125-174.

Goldstein, R., \& Vitevitch, M. S. (2014). The influence of clustering coefficient on wordlearning: How groups of similar sounding words facilitate acquisition. Frontiers in Psychology, 5. https://doi.org/10.3389/fpsyg.2014.01307

Hills, T. T., Maouene, M., Maouene, J., Sheya, A., \& Smith, L. (2009). Longitudinal analysis of early semantic networks. Psychological Science, 20(6), 729-739. https://doi.org/10.1111/j.1467-9280.2009.02365.x

Hsiao, Y., \& Nation, K. (2018). Semantic diversity, frequency and the development of lexical quality in children's word reading. Journal of Memory and Language, 103, 114-126.

Jaswal, V. K., \& Hansen, M. B. (2006). Learning words: Children disregard some pragmatic information that conflicts with mutual exclusivity. Developmental Science, 9(2), 158165.

Kuperman, V., Stadthagen-Gonzalez, H., \& Brysbaert, M. (2012). Age-of-acquisition ratings for 30,000 English words. Behavior Research Methods, 44(4), 978-990.

Kuznetsova, A., Brockhoff, P. B., \& Christensen, R. H. B. (2017). lmerTest package: Tests in linear mixed effects models. Journal of Statistical Software, 82(13).

Louwerse, M. M., \& Jeuniaux, P. (2010). The linguistic and embodied nature of conceptual processing. Cognition, 114(1), 96-104. 
FEATURE DISTINCTIVENESS 41

Love, B. C., Medin, D. L., \& Gureckis, T. M. (2004). SUSTAIN: A Network Model of Category Learning. Psychological Review, 111(2), 309-332. https://doi.org/10.1037/0033295X.111.2.309

Lund, K., \& Burgess, C. (1996). Producing high-dimensional semantic spaces from lexical cooccurrence. Behavior Research Methods, Instruments, \& Computers, 28(2), 203-208.

Markman, E. M., \& Wachtel, G. F. (1988). Children's use of mutual exclusivity to constrain the meanings of words. Cognitive Psychology, 20(2), 121-157.

Marques, J. F. (2005). Naming from definition: The role of feature type and feature distinctiveness. The Quarterly Journal of Experimental Psychology Section A, 58(4), 603-611. https://doi.org/10.1080/02724980443000106

McRae, K., Cree, G. S., Seidenberg, M. S., \& McNorgan, C. (2005). Semantic feature production norms for a large set of living and nonliving things. Behavior Research Methods, 37(4), $547-559$.

Minda, J. P., \& Smith, J. D. (2002). Comparing prototype-based and exemplar-based accounts of category learning and attentional allocation. Journal of Experimental Psychology: Learning, Memory, and Cognition, 28(2), 275.

Montefinese, M., Vinson, D., \& Ambrosini, E. (2018). Recognition memory and featural similarity between concepts: The pupil's point of view. Biological Psychology, 135, 159169.

Montefinese, M., Zannino, G. D., \& Ambrosini, E. (2015). Semantic similarity between old and new items produces false alarms in recognition memory. Psychological Research, 79(5), $785-794$. 
Moss, H. E., Tyler, L. K., \& Jennings, F. (1997). When leopards lose their spots: Knowledge of visual properties in category-specific deficits for living things. Cognitive Neuropsychology, 14(6), 901-950.

Moss, H. E., Tyler, L. K., \& Taylor, K. I. (2007). Conceptual structure. The Oxford Handbook of Psycholinguistics, 217-234.

Nelson, D. L., McEvoy, C. L., \& Schreiber, T. A. (2004). The University of South Florida free association, rhyme, and word fragment norms. Behavior Research Methods, Instruments, \& Computers, 36(3), 402-407.

Peters, R., \& Borovsky, A. (2019). Modeling early lexico-semantic network development: Perceptual features matter most. Journal of Experimental Psychology: General, 148(4), 763.

Pexman, P. M., Hargreaves, I. S., Siakaluk, P. D., Bodner, G. E., \& Pope, J. (2008). There are many ways to be rich: Effects of three measures of semantic richness on visual word recognition. Psychonomic Bulletin \& Review, 15(1), 161-167.

Pexman, P. M., Heard, A., Lloyd, E., \& Yap, M. J. (2017). The Calgary semantic decision project: Concrete/abstract decision data for 10,000 English words. Behavior Research Methods, 49(2), 407-417. https://doi.org/10.3758/s13428-016-0720-6

Pexman, P. M., Holyk, G. G., \& Monfils, M.-H. (2003). Number-of-features effects and semantic processing. Memory \& Cognition, 31(6), 842-855.

Pexman, P. M., Lupker, S. J., \& Hino, Y. (2002). The impact of feedback semantics in visual word recognition: Number-of-features effects in lexical decision and naming tasks. Psychonomic Bulletin \& Review, 9(3), 542-549. https://doi.org/10.3758/BF03196311 
Plaut, D. C., \& Shallice, T. (1993). Deep dyslexia: A case study of connectionist neuropsychology. Cognitive Neuropsychology, 10(5), 377-500. https://doi.org/10.1080/02643299308253469

Randall, B., Moss, H. E., Rodd, J. M., Greer, M., \& Tyler, L. K. (2004). Distinctiveness and correlation in conceptual structure: Behavioral and computational studies. Journal of Experimental Psychology: Learning, Memory, and Cognition, 30(2), 393.

Ratcliff, R., Gomez, P., \& McKoon, G. (2004). A Diffusion Model Account of the Lexical Decision Task. Psychological Review, 111(1), 159-182. https://doi.org/10.1037/0033295X.111.1.159

Recchia, G., \& Jones, M. (2012). The semantic richness of abstract concepts. Frontiers in Human Neuroscience, 6, 315.

Rips, L. J., Shoben, E. J., \& Smith, E. E. (1973). Semantic distance and the verification of semantic relations. Journal of Verbal Learning and Verbal Behavior, 12(1), 1-20.

Rodd, J., Gaskell, G., \& Marslen-Wilson, W. (2002). Making Sense of Semantic Ambiguity: Semantic Competition in Lexical Access. Journal of Memory and Language, 46(2), 245266. https://doi.org/10.1006/jmla.2001.2810

Siew, C. S. Q. (2019). spreadr: An R package to simulate spreading activation in a network. Behavior Research Methods, 1-20.

Siew, C. S. Q., \& Vitevitch, M. S. (2019). The phonographic language network: Using network science to investigate the phonological and orthographic similarity structure of language. Journal of Experimental Psychology: General, 148(3), 475-500. https://doi.org/10.1037/xge0000575 
Siew, C. S. Q., Wulff, D. U., Beckage, N. M., \& Kenett, Y. N. (2019). Cognitive Network Science: A review of research on cognition through the lens of network representations, processes, and dynamics. Complexity, 2019.

Sizemore, A. E., Karuza, E. A., Giusti, C., \& Bassett, D. S. (2018). Knowledge gaps in the early growth of semantic feature networks. Nature Human Behaviour, 2(9), 682.

Sloutsky, V. M., \& Fisher, A. V. (2004). Induction and Categorization in Young Children: A Similarity-Based Model. Journal of Experimental Psychology: General, 133(2), 166188. https://doi.org/10.1037/0096-3445.133.2.166

Smith, L. B., Jones, S. S., Landau, B., Gershkoff-Stowe, L., \& Samuelson, L. (2002). Object name learning provides on-the-job training for attention. Psychological Science, 13(1), $13-19$.

Steyvers, M., \& Tenenbaum, J. B. (2005). The large-scale structure of semantic networks: Statistical analyses and a model of semantic growth. Cognitive Science, 29(1), 41-78. https://doi.org/10.1207/s15516709 $\operatorname{cog} 2901 \_3$

Taylor, K. I., Devereux, B. J., Acres, K., Randall, B., \& Tyler, L. K. (2012). Contrasting effects of feature-based statistics on the categorisation and basic-level identification of visual objects. Cognition, 122(3), 363-374.

Taylor, K. I., Devereux, B. J., \& Tyler, L. K. (2011). Conceptual structure: Towards an integrated neurocognitive account. Language and Cognitive Processes, 26(9), 13681401.

Taylor, K. I., Salamoura, A., Randall, B., Moss, H., \& Tyler, L. K. (2008). Clarifying the nature of the distinctiveness by domain interaction in conceptual structure: Comment on Cree, McNorgan, and McRae (2006). 
Tousignant, C., \& Pexman, P. M. (2012). Flexible recruitment of semantic richness: Context modulates body-object interaction effects in lexical-semantic processing. Frontiers in Human Neuroscience, 6, 53.

Tyler, L. K., \& Moss, H. E. (2001). Towards a distributed account of conceptual knowledge. Trends in Cognitive Sciences, 5(6), 244-252.

Tyler, L. K., Moss, H. E., Durrant-Peatfield, M. R., \& Levy, J. P. (2000). Conceptual structure and the structure of concepts: A distributed account of category-specific deficits. Brain and Language, 75(2), 195-231.

Vinson, D. P., \& Vigliocco, G. (2008). Semantic feature production norms for a large set of objects and events. Behavior Research Methods, 40(1), 183-190.

Yap, M. J., Tan, S. E., Pexman, P. M., \& Hargreaves, I. S. (2011). Is more always better? Effects of semantic richness on lexical decision, speeded pronunciation, and semantic classification. Psychonomic Bulletin \& Review, 18(4), 742-750.

Zdrazilova, L., \& Pexman, P. M. (2013). Grasping the invisible: Semantic processing of abstract words. Psychonomic Bulletin \& Review, 20(6), 1312-1318. 


\section{Appendix A}

Correlations between predictors and outcome variables in the regression models.

Table A1. Age-of-acquisition norms $(N=4,013)$

\begin{tabular}{|c|c|c|c|c|c|c|c|}
\hline & Length & Frequency & Conc & NF & $\begin{array}{c}\text { Manhattan } \\
\text { distinctiveness }\end{array}$ & $\begin{array}{c}\text { Jaccard } \\
\text { distinctiveness }\end{array}$ & AoA \\
\hline Length & 1.00 & -0.36 & -0.21 & -0.14 & -0.10 & 0.07 & 0.39 \\
\hline Frequency & -0.36 & 1.00 & -0.14 & 0.05 & -0.03 & -0.08 & -0.57 \\
\hline Conc & -0.21 & -0.14 & 1.00 & 0.32 & 0.48 & -0.27 & -0.32 \\
\hline NF & -0.14 & 0.05 & 0.32 & 1.00 & 0.83 & -0.38 & -0.30 \\
\hline Manhattan distinctiveness & -0.10 & -0.03 & 0.48 & 0.83 & 1.00 & -0.39 & -0.30 \\
\hline Jaccard distinctiveness & 0.07 & -0.08 & -0.27 & -0.38 & -0.39 & 1.00 & 0.19 \\
\hline AoA & 0.39 & -0.57 & -0.32 & -0.30 & -0.30 & 0.19 & 1.00 \\
\hline
\end{tabular}

Table A2. Visual Lexical Decision (English Lexicon Project) $(N=4,013)$

\begin{tabular}{|c|c|c|c|c|c|c|c|c|c|c|}
\hline & Length & Frequency & AoA & ON & Conc & $\mathrm{NF}$ & $\begin{array}{c}\text { Manhattan } \\
\text { distinctiveness }\end{array}$ & $\begin{array}{c}\text { Jaccard } \\
\text { distinctiveness }\end{array}$ & $\begin{array}{c}\text { Z-scored } \\
\text { RT }\end{array}$ & $\begin{array}{c}\text { Mean } \\
\text { Accuracy }\end{array}$ \\
\hline Length & 1.00 & -0.36 & 0.39 & -0.65 & -0.21 & -0.14 & -0.10 & 0.07 & 0.52 & 0.00 \\
\hline Frequency & -0.36 & 1.00 & -0.57 & 0.31 & -0.14 & 0.05 & -0.03 & -0.08 & -0.64 & 0.39 \\
\hline AoA & 0.39 & -0.57 & 1.00 & -0.33 & -0.32 & -0.30 & -0.30 & 0.19 & 0.54 & -0.30 \\
\hline ON & -0.65 & 0.31 & -0.33 & 1.00 & 0.17 & 0.14 & 0.09 & -0.07 & -0.35 & 0.05 \\
\hline Conc & -0.21 & -0.14 & -0.32 & 0.17 & 1.00 & 0.32 & 0.48 & -0.27 & -0.04 & -0.06 \\
\hline $\mathrm{NF}$ & -0.14 & 0.05 & -0.30 & 0.14 & 0.32 & 1.00 & 0.83 & -0.38 & -0.11 & 0.03 \\
\hline $\begin{array}{l}\text { Manhattan } \\
\text { distinctiveness }\end{array}$ & -0.10 & -0.03 & -0.30 & 0.09 & 0.48 & 0.83 & 1.00 & -0.39 & -0.04 & 0.00 \\
\hline $\begin{array}{l}\text { Jaccard } \\
\text { distinctiveness }\end{array}$ & 0.07 & -0.08 & 0.19 & -0.07 & -0.27 & -0.38 & -0.39 & 1.00 & 0.10 & -0.04 \\
\hline
\end{tabular}


FEATURE DISTINCTIVENESS 47

\begin{tabular}{llllllllll} 
Z-scored RT & 0.52 & -0.64 & 0.54 & -0.35 & -0.04 & -0.11 & -0.04 & 0.10 & 1.00 \\
Mean Accuracy & 0.00 & 0.39 & -0.30 & 0.05 & -0.06 & 0.03 & 0.00 & -0.53 & 1.00 \\
\hline
\end{tabular}

Table A3. Semantic Decision (Calgary Semantic Project) $(N=1,336)$

\begin{tabular}{|c|c|c|c|c|c|c|c|c|c|c|}
\hline & Length & Frequency & AoA & Conc & ON & $\mathrm{NF}$ & $\begin{array}{c}\text { Manhattan } \\
\text { distinctiveness }\end{array}$ & $\begin{array}{c}\text { Jaccard } \\
\text { distinctiveness }\end{array}$ & $\begin{array}{c}\text { Z-scored } \\
\text { RT } \\
\end{array}$ & $\begin{array}{c}\text { Mean } \\
\text { Accuracy }\end{array}$ \\
\hline Length & 1.00 & -0.34 & 0.24 & 0.01 & -0.59 & -0.08 & 0.01 & -0.01 & 0.12 & 0.07 \\
\hline Frequency & -0.34 & 1.00 & -0.48 & -0.09 & 0.27 & 0.06 & -0.04 & -0.10 & -0.25 & 0.05 \\
\hline AoA & 0.24 & -0.48 & 1.00 & -0.43 & -0.21 & -0.35 & -0.36 & 0.23 & 0.53 & -0.25 \\
\hline Conc & 0.01 & -0.09 & -0.43 & 1.00 & 0.05 & 0.35 & 0.52 & -0.34 & -0.50 & 0.25 \\
\hline ON & -0.59 & 0.27 & -0.21 & 0.05 & 1.00 & 0.11 & 0.02 & -0.05 & -0.07 & -0.08 \\
\hline NF & -0.08 & 0.06 & -0.35 & 0.35 & 0.11 & 1.00 & 0.83 & -0.37 & -0.33 & 0.17 \\
\hline $\begin{array}{l}\text { Manhattan } \\
\text { distinctiveness }\end{array}$ & 0.01 & -0.04 & -0.36 & 0.52 & 0.02 & 0.83 & 1.00 & -0.40 & -0.41 & 0.24 \\
\hline $\begin{array}{l}\text { Jaccard } \\
\text { distinctiveness }\end{array}$ & -0.01 & -0.10 & 0.23 & -0.34 & -0.05 & -0.37 & -0.40 & 1.00 & 0.26 & -0.07 \\
\hline Z-scored RT & 0.12 & -0.25 & 0.53 & -0.50 & -0.07 & -0.33 & -0.41 & 0.26 & 1.00 & -0.66 \\
\hline Mean Accuracy & 0.07 & 0.05 & -0.25 & 0.25 & -0.08 & 0.17 & 0.24 & -0.07 & -0.66 & 1.00 \\
\hline
\end{tabular}

Legend:

AoA $=$ age of acquisition rating

Conc $=$ concreteness rating

$\mathrm{ON}=$ orthographic neighborhood size

$\mathrm{NF}=$ number of features

$\mathrm{RT}=$ reaction time $(z$-scored $)$ 


\section{Appendix B}

Computation of Jaccard and Manhattan distance measures.

Two different distance measures were computed to quantify the dissimilarity of any given pair of probability distributions: Jaccard distance and Manhattan distance. The first measure, Jaccard distance, is an example of a measure from inner-product family of distance measures that emphasizes shared information. Jaccard distance computes the distance between two words as subtracting the intersection of the two words' feature set distributions (i.e., a vector that represents the proportions of participants reporting each feature for a given word) over their union from 1. The second measure, Manhattan distance, is an example of a measure from the Minkowski family of distance measures that generally treats distance as the straight line between two points in Euclidean space. Manhattan distance computes the distance between two words as the sum of absolute differences between the proportions of participants reporting each feature. Mathematically, these measures were computed as follows: Jaccard distance, $d_{j}$ :

$$
d_{j}=1-\frac{\sum\left(P_{i} \times Q_{i}\right)}{\sum P_{i}^{2}+\sum Q_{i}^{2}-\sum\left(P_{i} \times Q_{i}\right)}
$$

Manhattan distance, $d_{m}$ :

$$
d_{m}=\sum\left|P_{i}-Q_{i}\right|
$$

where $P_{i}=\left[x_{1}, x_{2}, \ldots, x_{i}\right]$ is a vector representing the proportions of participants reporting each feature for a word 1 and $Q_{i}=\left[x_{1}, x_{2}, \ldots, x_{i}\right]$ is a vector representing the proportions of participants reporting each feature for a word 2. $i=$ the number of (unique) features in the feature production norms. 
Word-Feature Matrix

$\begin{array}{lrrrr} & \text { Feature 1 } & \text { Feature 2 } & \text { Feature 3 } & \text { Feature 4 } \\ \text { Word A } & 0.0 & 0.4 & 0.4 & 0.0 \\ \text { Word B } & 0.0 & 0.2 & 0.2 & 0.0 \\ \text { Word C } & 0.5 & 0.0 & 0.0 & 0.5\end{array}$

Jaccard distance network

Manhattan distance network

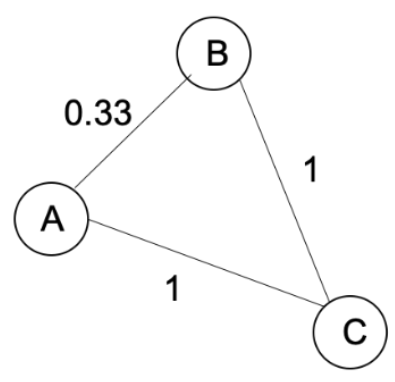

Distinctiveness

Word A Word B Word C

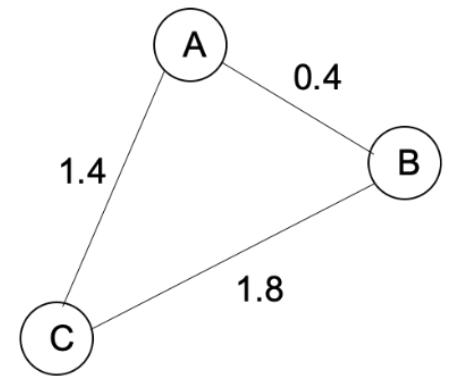

Distinctiveness

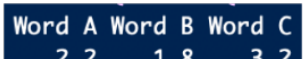

Figure B1. An example of two different distance measures used to compute feature

distinctiveness. Each word (A, B, and C) has a vector (i.e., row in the word-feature matrix) representing the proportion of individuals who reported each of the 4 features. Each feature is represented by a column in the word-feature matrix. Feature networks were computed for each distance measure such that nodes represented words and edges represented the distance between pairs of words. Feature distinctiveness for words are shown below each network. Words with greater values of distinctiveness are "further" from other nodes in the network. Adapted from Engelthaler and Hills (2017). 